\title{
Myocardial recruitment and the systolic efficiency slope; how do they relate to exercise performance in healthy young adults?
}

\author{
Jet van Zalen ${ }^{1}$, Sveeta Badiani ${ }^{1}$, Andrew d'Silva ${ }^{2}$, Anish Bhuva ${ }^{3}$, Nikhil Patel ${ }^{1}$, Alun \\ Hughes $^{4,5}$, Charlotte Manisty ${ }^{3}$, Sanjay Sharma ${ }^{2}$, James Moon ${ }^{3}$, and Guy Lloyd ${ }^{3}$ \\ ${ }^{1}$ Eastbourne District General Hospital \\ ${ }^{2}$ St George's University of London \\ ${ }^{3}$ Saint Bartholomew's Hospital Barts Heart Centre \\ ${ }^{4}$ University College London \\ ${ }^{5} \mathrm{UCL}$
}

June 22, 2020

\begin{abstract}
Aims The extent to which augmentation of heart function mirrors the increased metabolic demands of the peripheral musculature is not well characterised. The details of ventricular augmentation may provide insight into determinants of cardiac efficiency for optimal exercise performance. The aims were to establish how much of the variability in exercise performance could be explained by myocardial recruitment, and which parameter of systolic function was most closely related to exercise performance. Methods and results Untrained volunteers were recruited prior to training for the London Marathon. All performed a cardiopulmonary exercise test combined with stress echocardiography. Systolic and diastolic longitudinal velocities (S' and E'), ejection fraction (LVEF), stroke volume (SV) and strain were obtained throughout exercise. Continuous S' showed a strong correlation with absolute VO2 (rho=0.83; $<<0.0001)$. Only SV and S' were predictive of VO2peak. LVEF and E' as well as both global longitudinal and circumferential strain showed no correlation. The systolic efficiency slope (SES) was calculated by determining the individual regression lines for $\mathrm{VO} 2$ and S'. A moderate relationship between the SES and VO2peak was observed for both septal S'; r=0.57; $<0.001$ and lateral S', r=0.53; $<<0.001$ ). Conclusion A detailed description of myocardial function is described; linear for S' and E' and a plateau for EF and GLS. S' during exercise is a better predictor of exercise performance than LVEF, SV or GLS. The SES slope was able to predict VO2peak suggesting the process driving systolic velocity and its augmentation is a key determinant of exercise ability.
\end{abstract}

\section{Keywords}

Cardiopulmonary exercise test

Stress echocardiography

Myocardial augmentation slope

\section{Introduction}

The relationship between resting echocardiographic measures of cardiac function and exercise capacity is weak ${ }^{1-4}$. At peak exercise, left ventricular (LV) function, especially longitudinal motion, is better correlated with maximum exercise capacity ${ }^{5}$. The shape of the relationship, as well as the relative contribution of various measurable facets of heart function is not known. Amongst athletes with phenotypically abnormal hearts, augmentation of left ventricular ejection fraction (LVEF) by more than $10 \%$ differentiates those with adaptive rather than cardiomyopathic hearts ${ }^{6,7}$. Understanding the shape to the relationship between contractile 
reserve and exercise attainment is vital to understand the physiology, and to establish target parameters that might be useful when investigating disease states. We therefore hypothesized that detailed measures of exercise myocardial recruitment would explain individual exercise performance, and that longitudinal function would be the primary adaptive strategy. This was achieved by recording oxygen uptake $\left(\mathrm{VO}_{2}\right)$ and echocardiography simultaneously across an incremental exercise protocol in a cohort of healthy volunteers.

\section{Methods}

Full details of the protocol have been previously published ${ }^{8}$ and this was a pre-planned ancillary analysis. Healthy participants were recruited in 2016 prior to undertaking training their first marathon (Virgin Money London). Inclusion criteria age was less than 35 years at recruitment, no past significant medical history and no previous marathon running experience. All procedures were in accordance with the principles of the Helsinki declaration. All participants gave written informed consent and the study was approved by the London-Queen Square National Research Ethics Service Committee (15/LO/0086).

All measurements were conducted before training started over a three-week period (three consecutive weekends after ballot place announcement), six months prior to the marathon.

\section{Data acquisition and analysis}

Cardiopulmonary exercise testing (CPET) was performed according to exercise testing guidelines ${ }^{9}$. Protocols were individually determined with work rate $(15-30 \mathrm{~W})$ increased every minute until voluntary exhaustion, aiming for 10 minutes of exercise. To permit concurrent transthoracic echocardiography, studies were performed using a semi-recumbent cycle ergometer (ERG $911 \mathrm{~S} / \mathrm{L}$, Schiller, Baar, Switzerland). A 1-minute rest period was included followed by a 3-minute warm up. Heart rate (HR), blood pressure and oxygen saturation were monitored throughout. $\mathrm{VO}_{2}$ was continuously measured using a calibrated breath-by-breath analyser (Cosmed Quark CPET, Rome, Italy). Participants were verbally encouraged to exercise until maximal exertion. $\mathrm{VO}_{2}$ peak was expressed as the highest value from an average of 30 seconds during the final stage of the exercise test. To fully assess submaximal efforts, the oxygen uptake efficiency slopes (OUES) was calculated automatically from $\mathrm{VO}_{2}$ against the logarithm of $\mathrm{V}_{\mathrm{E}}\left(\log \mathrm{V}_{\mathrm{E}}\right)^{10}$.

Echocardiography was performed using a GE Vivid E95 platform (Vingmed-General Electric, Horten, Norway) equipped with a phased-array transducer (1.4-4.6 MHz). A detailed protocol was collected at rest, at 5 minutes into exercise (excluding the 3-minute warm-up period), and when the respiratory exchange ratio (RER) was above 1.0. This included the apical 4-chamber view (with and without tissue velocity imaging (TVI), the apical two-chamber, apical long axis view, parasternal short axis view at the base and apical level, and a PW at the level of the Left ventricular outflow tract (LVOT) (one centimetre below the aortic valve). An abbreviated protocol was obtained every 1 minute and 15 seconds and included the apical 4-chamber view (with and without TVI). As part of the protocol 12-lead ECG and cardiac magnetic resonance was undertaken but not included in this analysis.

\section{Echocardiographic analysis}

All analyses were carried out offline using the GE Echopac software (version 113). S' was derived from colourderived TVI images where the sample volume was placed at the annulus of the septal and lateral walls. S' was defined as the highest velocity during systole after the end of isovolumetric contraction. Three cardiac cycles were obtained and S' was averaged for each time point. During the full protocol Simpson's biplane was measured (in the apical 4-and 2-chamber view), global longitudinal strain (GLS) in the three apical views and the LVOT velocity time integral (VTI). Speckle-tracking strain analysis was accepted when the software and visual inspection indicated adequate tracking. If tracking was inadequate, manual adjustments were made or the analysis was excluded from overall analyses. GLS was obtained from the apical 4-chamber view (basal septum, mid septum, apical septum, basal lateral, mid lateral, and apical lateral), the apical 2-chamber view (basal inferior, mid inferior, apical inferior, basal anterior, mid anterior, apical anterior) and the apical long axis view (basal posterior, mid posterior, basal anteroseptal, mid anteroseptal). If data was 
of insufficient quality it was excluded from analyses. This was defined as poor TVI tracing, exclusion of the mitral valve annulus (for colour TVI measurements), or poor endocardial border definition (for biplane). Eleven participants were excluded from analysis.

\section{Statistical analyses}

All data are expressed as mean \pm standard deviation (SD) for continuous variables. Categorical data are expressed as absolute values and percentages. All data was tested for normality using the KolmogorovSmirnov statistical test. Pearson's correlation coefficients (r) or Spearman's coefficients (rho) were used to determine relationships between haemodynamic and echocardiographic responses. $\mathrm{VO}_{2}$ was averaged every 10 seconds and were linked with corresponding S' values every minute. E', LVEF, GLS and circumferential strain values were linked with corresponding S' values at rest, 5 minutes and when the RER was over 1 . Values at rest, at 5 minutes and when RER was over 1 were compared using one-way ANOVA and Tukey post-hoc analyses to determine differences between groups. A scatter plot with $95 \%$ confidence interval was plotted. The augmentation curve was investigated in more detail (LVEF, systolic and diastolic volumes, LVOT VTI and GLS) and correlated with corresponding $\mathrm{VO}_{2}$ peak values. Inter- and intraobserver measurement variability was performed for 5 randomly selected participants for echocardiographic parameters and for 15 randomly selected participants for CPET parameters. Two BSE accredited operators (JVZ, SB) with extensive experience in performing CPET simultaneously with echocardiography performed the inter- and intra-observer measurements. The intraclass correlation coefficient (ICC) and 95\% confidence intervals was used to quantify reproducibility. All statistical analyses were performed using the Statistical Package for the Social Sciences (SPSS version 20.0; SPSS, Inc). A P-value of $<0.05$ was considered statistically significant.

\section{Results}

\section{Population}

Of the 68 patients recruited 11 were excluded (nine studies were off insufficient image quality for meaningful analysis and 2 studies did not have TDI switched on during exercise). Fifty-seven participants were included in the final analysis. Thirty-two $(56 \%)$ were male, aged $29.2 \pm 3.3$ years, weight of $71.6 \pm 12.9 \mathrm{~kg}$ and height of $175 \pm 10.3 \mathrm{~cm}$. Baseline LVEF, end-diastolic (EDV) and end-systolic volumes (ESV) were within normal ranges (LVEF: $61.0 \pm 2.8 \%$; EDV: $120 \pm 30.2 \mathrm{~mL}$; ESV; $46.9 \pm 13.3 \mathrm{~mL}$ ) (table 1 and 2).

\section{Exercise performance}

Peak RER was $1.23 \pm 0.09$ with $56(98 \%)$ of participants achieving an RER of $>1.10$ (1 participant reached a peak RER of 1.08). Absolute $\mathrm{VO}_{2}$ peak was $2.8 \pm 0.7 \mathrm{~L} \cdot \mathrm{min}^{-1}$ and relative $\mathrm{VO}_{2}$ peak was $38.7 \pm 6.5$ $\mathrm{mL} \cdot \mathrm{kg}^{-1} \cdot \mathrm{min}^{-1}$. OUES was $2936 \pm 749\left(\mathrm{~mL} \cdot \mathrm{min}^{-1}\right)^{*}\left(\mathrm{~L}^{*} \mathrm{~min}^{-1}\right)^{-1}($ table 1$)$.

\section{Mechanical augmentation}

All parameters with the exception of EDV significantly augmented after 5 minutes of exercise (table 2). EF increased by $13+-7.2 \%$, stroke volume by $22+-17 \%$, GLS by $22+-15 \%$ and septal S' and lateral S' by $52+-24$ and $61+-27 \%$ respectively. At RER $>1$ only longitudinal velocities (systolic and diastolic, septal and lateral) continued to show statistically significant augmentation. All other parameters demonstrated an augmentation plateau (Table 2; Figure 1).

\section{Longitudinal contractile reserve - $\mathrm{VO}_{2}$ relationship}

The augmentation of longitudinal velocity showed a strong correlation with $\mathrm{VO}_{2}$ throughout exercise for the septal S' $(\mathrm{rho}=0.86, \mathrm{p}<0.0001)$, the lateral $\mathrm{S}$ ' $(\mathrm{rho}=0.78, \mathrm{p}<0.0001)$ and for the average $\mathrm{S}$ ' (rho=0.83, $\mathrm{p}<0.0001$ ) throughout exercise (Figure 2). Diastolic velocities also demonstrated a good relationship with $\mathrm{VO}_{2}$ (septal E'; rho=0.8, $\mathrm{p}<0.0001$; lateral E'; rho=0.78) (Table 2). The relationship was stronger with the septum than the lateral wall (Table 3 ).

On multivariate testing only stroke volume and systolic velocities (especially at RER $>1$ in the septum) were predictive of $\mathrm{VO}_{2}$ peak. Diastolic parameters, ejection fraction as well as both global longitudinal and 
circumferential strain showed no correlation with $\mathrm{VO}_{2}$ peak (Table 4).

\section{Systolic efficiency slope (SES) - the $\mathrm{S}^{\prime} / \mathrm{VO}_{2}$ relationship}

We took advantage of the linear augmentation pattern of systolic velocity and its close relationship with $\mathrm{VO}_{2}$ to derive individual regression lines for $\mathrm{VO}_{2}$ and $\mathrm{S}^{\prime}$. This new parameter represents the amount of S' associated with a 1 unit increase in $\mathrm{VO}_{2}$ and therefore a measure of myocardial efficiency. A relationship between the SES slope was observed for $\mathrm{VO}_{2}$ peak and septal $\mathrm{S}$; $\mathrm{r}=0.57 ; \mathrm{p}<0.001$ and lateral $\mathrm{S}$; $\mathrm{r}=$ $0.53 ; \mathrm{p}<0.001$ ) (Figure 3 ). We also confirmed the validity of these findings by creating models for OUES, a submaximal measures shown to predict total cardiopulmonary capacity ${ }^{10,11}$. A significant relationship was found between SES slope and the OUES (septal $\mathrm{r}=0.54 ; \mathrm{p}<0.001$, lateral $\mathrm{r}=0.52 ; \mathrm{p}=0.001$ ).

\section{Inter- and intraobserver reproducibility}

There was excellent inter- and intra-observer reproducibility for all measurements by CPET $\left(\mathrm{VO}_{2}\right.$ peak and OUES). For echocardiography parameters there was good to excellent inter- and intraobserver reproducibility for S' and E' at rest and exercise. There was lower inter- and intraobserver reproducibility seen for LVEF and GLS both at rest and exercise (Appendix 1).

\section{Discussion}

There are significant gaps in the understanding of myocardial augmentation and its relationship with exercise performance. This study addresses these by demonstrating that systolic and (to a lesser extent) diastolic myocardial velocities increase incrementally as exercise work rate increases in a linear fashion, in contrast to parameters that are measured throughout systole (LVEF, SV, GLS) which show a plateau after low intensity exercise. We provide ranges for the expected augmentation in healthy young adults both at low intensity and at peak exercise. We demonstrate that systolic velocities are very closely linked to $\mathrm{VO}_{2}$ throughout exercise and, in our study, systolic velocities during exercise are a better predictor of exercise performance than LVEF, SV or GLS; and we define a new parameter (SES) equivalent to the slope of this relationship that can predict exercise capacity.

Although the augmentation of normal and pathological heart function has been measured this is usually at the start, at some fixed point, during or after the cessation of exercise. This makes comparison complex. We found a $14 \%$ increase in LVEF during exercise with the majority of the increase happening during the initial bout of exercise which is very comparable with the existing literature which suggests that LVEF beyond the ventilatory threshold show a slightly plateau response ${ }^{12}$. In normal individuals, in an upright position, EDV slightly increases with incremental exercise, with either no change or small decrease in ESV ${ }^{13,14}$. However body position effects the change in EDV and ESV during exercise. In a recumbent position EDV remains unchanged during exercise and an increase in seen in ESV ${ }^{15}$. We used a semi-recumbent cycle ergometer and found a similar response to volumes.

GLS is a more advanced method of measuring LV function. Wang et al.(2014) ${ }^{16}$ showed a significantly increase in GLS and S' during exercise in healthy control patients, our data showed a $25 \%$ increase in GLS and $89 \%$ increase in S', which is a similar. However GLS is more problematic to measure at peak exercise because high heart rates may lead to speckle misregistration, although anecdotally higher than recommended heart rates are tolerated by the software. Cifra et al. , (2016) described a linear relationship between HR and GLS during exercise and showed that S' obtained using colour Doppler tissue imaging showed excellent inter-and intra-observer variability. GLS measurements were more challenging to obtain during exercise due to lung interference and more excessive cardiac motion. However although peak systolic strain values showed higher interobserver variability it was still considered acceptable for clinical use ${ }^{17}$.

There is limited data on the relationship between myocardial augmentation and exercise performance. We have previously published that peak systolic velocity and $\mathrm{VO}_{2}$ are closely correlated across a very wide range of diagnoses ${ }^{5,18}$. This data, while an interesting proof of concept, does not resolve the question of whether the heart is behaving differently, or whether those with less severe disease are simply achieving more exercise and hence more myocardial augmentation. This central weakness, the confounding effect of exercise ability, 
runs through much of the contractile reserve literature. Attempts to resolve this by imaging at submaximal exercise are vulnerable to an incomplete understanding of the pattern of augmentation for each measured parameter.

The use of a semi-recumbent cycle ergometer allows the combination with CPET and echocardiography to be combined, providing additional insight into any observed changes in heart function. There have been limited attempts to relate changes on myocardial function on echocardiography with changes in $\mathrm{VO}_{2}$. In a study of 31 patients (12 with HF, 15 with preserved LVEF and 16 with reduced LVEF and 15 controls); they demonstrated an increase in S' and E' from baseline, to the ventilatory threshold up to maximal effort, a result similar to the data in a healthy cohort. GLS and LVEF also augmented but the relationship was weaker, as did the additional measures of the RV and circumferential strain ${ }^{19}$. Resting LVEF is not able to predict $\mathrm{VO}_{2}$ peak ${ }^{4,20,21}$. However obtaining LVEF during exercise does have incremental prognostic value. HF patients who are able to increase LVEF by more than $5 \%$ have a better prognosis than those we do not 22. Evaluating GLS at rest is an independent and incremental prognostic tool regarding long-term risk in cardiovascular morbidity and mortality ${ }^{23}$. However GLS at exercise requires more investigation.

In our study, we selected S' as our principle long axis evaluation parameter as it is a reproducible measurement during exercise ${ }^{2}$. Mechanistically longitudinal S' has previously shown to be an early indicator for ventricular dysfunction, as it is one of the principle engines of systole, and a fall often precedes a subsequent fall in $\mathrm{LVEF}^{24}$ during disease; unlike other parameters of both volumetric and longitudinal function $\mathrm{S}$ ' augmentation remained linear during exercise. In the analysis S' was more predictive of $\mathrm{VO}_{2}$ peak than EF or GLS both at 5 minutes of exercise, when all parameters showed augmentation and at peak exercise, when EF and GLS had plateaued. It is easily forgotten that strain and velocity measure different aspects of systolic performance and cannot be considered interchangeable. Work by Gu et al. (2017) looking at the first phase EF, during which with, normal activation, peak velocity is reached, has demonstrated that within systole the initial phase of contraction is the most important ${ }^{25}$. This provides a rationale for the superiority of systolic velocity over the whole systolic measures EF and GLS. Our study confirms the very tight relationship between $\mathrm{VO}_{2}$ and $\mathrm{S}$ ' throughout the whole of exercise, making it a very useful surrogate for $\mathrm{VO}_{2}$ and suggesting that the processes that increase myocardial velocity are central to determine exercise function.

By inference therefore variation in, or changes to, cardiac function would result in different relationships between $\mathrm{VO}_{2}$ and S'. This is important because of the potential that augmentation of S' is simply reflecting a longer exercise time or greater workload making the relationship tautological. To understand the relationship between $\mathrm{VO}_{2}$ and $\mathrm{S}^{\prime}$ in greater detail we hypothesised that the individual ratios of $\mathrm{S}^{\prime}$ to $\mathrm{VO}_{2}$ (the amount of myocardial augmentation required to increase $\mathrm{VO}_{2}$ by a single unit) would be able to predict $\mathrm{VO}_{2}$ peak values. We devised the term the Systolic Efficiency Slope (SES) to describe this. There was a relationship between the SES and $\mathrm{VO}_{2}$ peak suggesting that this relationship may be important. But given that peak exertion can be heavily effort dependent we also looked at OUES (a well validated submaximal measure known to be highly related to $\mathrm{VO}_{2}$ peak). Again there was a significant relationship. This contraction / metabolic coupling relationship has not been previously described in this way. The SES slope; a slope that does not require a pre-specified heart rate or maximal exercise to be achieved. While the data is not strong enough to suggest this as a clinical tool, we propose these may be suitable methods for future research.

\section{Limitations}

Our population was young and verified to be without cardiac disease and despite this 11 patients were excluded mainly due to insufficient image quality. Only participants with analysable image quality were included. The imaging protocol was arduous, however the findings (threshold values for EF and GLS / continuous augmentation of $S^{\prime}$ ) would allow simplification in future studies that would also be more clinically achievable. Non invasive blood pressure was measured as part of the protocol, however the sampling from the automatic detection devices was consistently poor resulting in less than $50 \%$ evaluable data. Analysis requiring blood pressure was therefore not performed. These findings may not apply to a disease population or those from other age groups. TVI measurements were optimised for perpendicular septal measurements. Lateral wall TVI may have been off axis which can introduce error. Transmitral Doppler was not recorded 
because of the technical challenge of obtaining these values at high rate show complete E/A fusion making detection of the true Doppler E wave and hence an unreliable E/E'. The GLS measured by speckle tracking may not be accurate at the higher heart rate. Our data would have permitted the evaluation of myocardial work, but this did not form part of the original protocol and is vendor specific, therefore this analysis has not been undertaken.

\section{Conclusion}

We have described in detail the augmentation of a variety of systolic parameters during exercise in fit young adults demonstrating two patterns of augmentation; linear for S' and E' and a plateau for EF and GLS. The magnitude of the augmentation ( $14 \%$ for $\mathrm{EF} / 89 \%$ for S' is in line with previous research. The study describes the relationship between longitudinal function (as expressed by $\mathrm{S}^{\prime}$ ) and $\mathrm{VO}_{2}$ throughout exercise. Systolic velocity, which represents early and mid-systolic function, is more important than whole systolic parameters such as ejection fraction including GLS in predicting exercise performance. The SES described for the first time shows that the regressive relationship between $S^{\prime} / \mathrm{VO}_{2}$ predicts peak exercise tolerance which proves that the relationship is more than simply collinearity. It also offers a novel research opportunity to evaluate disease states where this relationship may be different. We abandon the past at our peril, and this data suggests a move from velocity to strain may not be justified or helpful in evaluating myocardial performance during exercise.

Acknowledgements

We thank the study participants for their time and taking part in the study. We are also grateful to Virgin London Marathon for their support in recruitment of participants. We are grateful to the entire marathon study team performing investigations. In addition to the authors of this manuscript, The Marathon Study group included the following staff from St George's University of London, University College London, Bart's Health Trust and other organizations.

\section{Funding}

The Marathon Study was funded by the British Heart Foundation (FS/15/27/31465), Cardiac Risk in the Young, and the Barts Cardiovascular Biomedical Research Centre. The study received support from COSMED (Rome, Italy) through the provision of cardiopulmonary exercise testing equipment and technical support.

\section{References}

1. Witte KKA, Nikitin NP, De Silva R, et al. Exercise capacity and cardiac function assessed by tissue Doppler imaging in chronic heart failure. Heart 2004;90 :1144-50.

2. Rubis P, Podolec P, Tomkiewicz-Pajak L, et al. Usefulness of the Evaluation of Isovolumic and Ejection Phase Myocardial Signals during Stress Echocardiography in Predicting Exercise Capacity in Heart Failure Patients. Echocardiography 2009;26:1050-9.

3. Clark AL, Swan JW, Laney R, et al. The role of right and left ventricular function in the ventilatory response to exercise in chronic heart failure. Circulation 1994;89 :2062-9.

4. Carell ES, Murali S, Schulman DS, et al. . Maximal exercise tolerance in chronic congestive heart failure : Relationship to resting left ventricular function. Chest 1994;106 :1746-52.

5. McIntosh RA, Silberbauer J, Veasey RA, et al. Tissue Doppler-Derived Contractile Reserve Is a Simple and Strong Predictor of Cardiopulmonary Exercise Performance across a Range of Cardiac Diseases.Echocardiography 2013;30:527-33.

6. Millar Lynne, Fernandez Gabriel, Dhutia Harshil, et al.Abstract 15662: Exercise Echocardiography Has a High Sensitivity and Specificity in Differentiating Athlete's Heart From Dilated Cardiomyopathy. Circulation 2016;134 :A15662-A15662. 
7. Claessen G, Schnell F, Bogaert J, et al. Exercise cardiac magnetic resonance to differentiate athlete's heart from structural heart disease. Eur Heart J Cardiovasc Imaging2018;19 :1062-70.

8. Jones S, D'Silva A, Bhuva A, et al. Improved Exercise-Related Skeletal Muscle Oxygen Consumption Following Uptake of Endurance Training Measured Using Near-Infrared Spectroscopy. Front Physiol2017;8

9. Gibbons RJ, Balady GJ, Bricker JT, et al. ACC/AHA 2002 Guideline Update for Exercise Testing: Summary Article A Report of the American College of Cardiology/American Heart Association Task Force on Practice Guidelines (Committee to Update the 1997 Exercise Testing Guidelines). Circulation 2002;106 :1883-92.

10. Baba R, Nagashima M, Goto M, et al. Oxygen uptake efficiency slope: a new index of cardiorespiratory functional reserve derived from the relation between oxygen uptake and minute ventilation during incremental exercise. J Am Coll Cardiol1996;28:1567-72.

11. Hollenberg M, Tager IB. Oxygen uptake efficiency slope: an index of exercise performance and cardiopulmonary reserve requiring only submaximal exercise. J Am Coll Cardiol 2000;36:194-201.

12. Rodrigues EA, Maddahi J, Brown H, et al. Responses of left and right ventricular ejection fractions to aerobic and anaerobic phases of upright and supine exercise in normal subjects. American Heart Journal $1989 ; 118: 319-24$.

13. Rubal BJ, Moody JM, Damore S, et al. Left ventricular performance of the athletic heart during upright exercise: a heart rate-controlled study. Medicine 63 Science in Sports $\mathcal{E}$ Exercise1986;18 :134-139.

14. Brynjolf I, Kelbaek H, Munck O, et al. . Right and left ventricular ejection fraction and left ventricular volume changes at rest and during exercise in normal subjects. Eur Heart J1984;5 :756-61.

15. Mizuno H, Yanagisawa A, Shigeyama T, et al. Continuous ambulatory radionuclide monitoring of left ventricular function: effect of body position during ergometer exercise. J Nucl Med1997;38:1669-72.

16. Wang J, Fang F, Wai-Kwok Yip G, et al. Changes of ventricular and peripheral performance in patients with heart failure and normal ejection fraction: insights from ergometry stress echocardiography.Eur $J$ Heart Fail 2014;16 :888-97.

17. Cifra B, Mertens L, Mirkhani M, et al. Systolic and Diastolic Myocardial Response to Exercise in a Healthy Pediatric Cohort. J Am Soc Echocardiogr 2016;29:648-54.

18. Zalen J van, Patel NR, Podd SJ, et al. Prognostic importance of tissue velocity imaging during exercise echocardiography in patients with systolic heart failure. Echo Res Pract2015;2 :19-27.

19. Shimiaie J, Sherez J, Aviram G, et al. Determinants of Effort Intolerance in Patients With Heart Failure. JACC: Heart Failure 2015;3 :803-14.

20. Benge W, Litchfield RL, Marcus ML. Exercise capacity in patients with severe left ventricular dysfunction. Circulation 1980;61:955-9.

21. Cohn JN, Johnson GR, Shabetai R, et al. Ejection fraction, peak exercise oxygen consumption, cardiothoracic ratio, ventricular arrhythmias, and plasma norepinephrine as determinants of prognosis in heart failure. The V-HeFT VA Cooperative Studies Group.Circulation 1993;87 :VI5-16.

22. Pedone C, Bax JJ, van Domburg RT, et al. Long-term prognostic value of ejection fraction changes during dobutamine-atropine stress echocardiography. Coron Artery Dis 2005;16 :309-13.

23. Biering-Sorensen T, Biering-Sorensen SR, Olsen FJ, et al.Global Longitudinal Strain by Echocardiography Predicts Long-Term Risk of Cardiovascular Morbidity and Mortality in a Low-Risk General Population: The Copenhagen City Heart Study. Circ Cardiovasc Imaging 2017;10 . 
24. Yu C-M, Sanderson JE, Marwick TH, Oh JK. Tissue Doppler Imaging: A New Prognosticator for Cardiovascular Diseases. Journal of the American College of Cardiology 2007;49 :1903-14.

25. Gu H, Li Y, Fok H, et al. Reduced First-Phase Ejection Fraction and Sustained Myocardial Wall Stress in Hypertensive Patients With Diastolic Dysfunction: A Manifestation of Impaired Shortening Deactivation That Links Systolic to Diastolic Dysfunction and Preserves Systolic Ejection Fraction. Hypertension 2017;69 :633-40.

Table 1: Baseline characteristics

\begin{tabular}{ll}
\hline & All participants $(\mathrm{n}=57)$ \\
\hline Height $(\mathrm{cm})$ & $175 \pm 10.3$ \\
Weight $(\mathrm{kg})$ & $71.6 \pm 12.9$ \\
Age $($ years $)$ & $29.2 \pm 3.3$ \\
Male $(\%)$ & $32(56 \%)$ \\
$\mathrm{VO}_{2}$ peak $\left(\mathrm{L} \cdot \mathrm{min}^{-1}\right)$ & $2.8 \pm 0.7$ \\
$\mathrm{VO}_{2}$ peak $\left(\mathrm{mL} \cdot \mathrm{kg}^{-1} \cdot \mathrm{min}^{-1}\right)$ & $38.7 \pm 6.5$ \\
Predicted VO$(\%)$ & $107 \pm 17$ \\
WR peak $(\mathrm{Watts})$ & $226 \pm 55$ \\
HR peak $\left(\mathrm{beats} \cdot \mathrm{min}^{-1}\right)$ & $168 \pm 17.5$ \\
Peak RER & $1.23 \pm 0.09$ \\
OUES $\left(\mathrm{mL} \cdot \mathrm{min}^{-1}\right) *\left(\mathrm{~L}^{*} \mathrm{~min}^{-1}\right)^{-1}$ & $2936 \pm 749$ \\
\hline
\end{tabular}

$\mathrm{VO}_{2}$ peak- peak oxygen uptake; WR - work rate; HR - heart rate; RER - Respiratory Exchange Ratio; OUES - oxygen uptake efficiency slope

Table 2: Haemodynamic and echocardiographic responses at baseline and exercise (mean \pm SD)

\begin{tabular}{llllll}
\hline & Rest & 5 minutes & RER $>1.0$ & \% change from rest to 5 minutes & \% change from rest \\
\hline Septal S' $\left(\mathrm{cm} \cdot \mathrm{s}^{-1}\right)$ & $7.2 \pm 1.4$ & $11.3 \pm 1.8^{*}$ & $13.7 \pm 1.8^{\S}$ & $61 \pm 27$ & $96 \pm 45$ \\
Lateral S' $\left(\mathrm{cm} \cdot \mathrm{s}^{-1}\right)$ & $8.4 \pm 1.6$ & $12.5 \pm 1.6^{*}$ & $14.9 \pm 1.7^{\S}$ & $52 \pm 24$ & $81 \pm 39$ \\
Septal E' $\left(\mathrm{cm} \cdot \mathrm{s}^{-1}\right)$ & $10.1 \pm 1.4$ & $15.3 \pm 2.8^{*}$ & $18.1 \pm 2.7^{\S}$ & $54 \pm 29$ & $78 \pm 31$ \\
Lateral E' $\left(\mathrm{cm} \cdot \mathrm{s}^{-1}\right)$ & $12.8 \pm 2.0$ & $17.0 \pm 2.7^{*}$ & $18.8 \pm 2.5^{\S}$ & $35 \pm 23$ & $49 \pm 31$ \\
LVEF $(\%)$ & $61.0 \pm 2.8$ & $68.8 \pm 3.6^{*}$ & $69.9 \pm 3.9$ & $13 \pm 7.2$ & $14 \pm 7.5$ \\
EDV $(\mathrm{mL})$ & $120 \pm 30.2$ & $113 \pm 38.0$ & $112 \pm 25.8$ & $-2.1 \pm 14$ & $-6 \pm 15$ \\
ESV $(\mathrm{mL})$ & $46.9 \pm 13.3$ & $36.7 \pm 11.5^{*}$ & $34.3 \pm 9.2$ & $-21 \pm 14$ & $-25 \pm 16$ \\
SV (mL) & $67.4 \pm 14.9$ & $81.1 \pm 17.0^{*}$ & $84.6 \pm 19.1$ & $22 \pm 17$ & $27 \pm 18$ \\
HR (beats $\cdot$ min $\left.^{-1}\right)$ & $68.6 \pm 10.6$ & $130 \pm 14.8^{*}$ & $151 \pm 14.0^{\S}$ & $93 \pm 32$ & $128 \pm 34$ \\
GLS (\%) & $-18.0 \pm 2.4$ & $-21.4 \pm 3.1^{*}$ & $-21.8 \pm 2.7$ & $-22 \pm 15$ & $-25 \pm 26$ \\
Circumferential strain $(\%)$ & $-20.1 \pm 4.7$ & $-23.2 \pm 4.3$ & $-23.3 \pm 4.3$ & $-16 \pm 25$ & $-16 \pm 25$ \\
\hline
\end{tabular}

* Baseline vs. 5 minutes; $\mathrm{p}<0.05 ; \S ; 5$ minutes vs. RER $>1.0 ; \mathrm{p}<0.05$

HR - heart rate; S' - systolic velocity; E' - diastolic velocity; LVEF - left ventricular ejection fraction; EDV - end diastolic volume; ESV- end systolic volume; GLS - global longitudinal strain; SV - stroke volume

Table 3: Correlations between $\mathrm{V0}_{2}$ measures of myocardial function throughout exercise

\begin{tabular}{ll}
\hline & $\mathrm{VO}_{2}\left(\mathrm{~L} \cdot \mathrm{min}^{-1}\right)$ \\
\hline Sep S' $\left(\mathrm{cm} \cdot \mathrm{s}^{-1}\right)$ & $0.86^{*}$
\end{tabular}




\begin{tabular}{ll}
\hline & $\mathrm{VO}_{2}\left(\mathrm{~L} \cdot \mathrm{min}^{-1}\right)$ \\
\hline Lat S' $\left(\mathrm{cm} \cdot \mathrm{s}^{-1}\right)$ & $0.80^{*}$ \\
Sep E' $\left(\mathrm{cm} \cdot \mathrm{s}^{-1}\right)$ & $0.78^{*}$ \\
Lat E' $\left(\mathrm{cm} \cdot \mathrm{s}^{-1}\right)$ & $0.70^{*}$ \\
LVEF $(\%)$ & $0.66^{*}$ \\
ESV $(\mathrm{mL})$ & -0.17 \\
SV $(\mathrm{mL})$ & 0.56 \\
GLS $(\%)$ & -0.46 \\
\hline
\end{tabular}

${ }^{*} \mathrm{P}<0.05$

S' - Systolic longitudinal velocity; E' - diastolic velocity; $\dot{V}_{2}$ - oxygen uptake; LVEF - Left ventricular ejection fraction; ESV - end systolic volume; SV - stroke volume; GLS - global longitudinal strain

Table 4: Correlations between measures of myocardial function and $\mathrm{VO}_{2}$ peak at baseline low intensity and peak intensity exercise

\begin{tabular}{llll}
\hline & $\mathrm{VO}_{2}$ peak $\left(\mathrm{L} \cdot \mathrm{min}^{-1}\right)$ & $\mathrm{VO}_{2}$ peak $\left(\mathrm{L} \cdot \mathrm{min}^{-1}\right)$ & $\mathrm{VO}_{2}$ peak $\left({\left.\mathrm{L} \cdot \mathrm{min}^{-1}\right)}\right.$ \\
\hline & Rest & $5 \mathrm{~min}$ & $\mathrm{RER}>1$ \\
Sep S' $\left(\mathrm{cm} \cdot \mathrm{s}^{-1}\right)$ & $0.48^{*}$ & $0.27^{*}$ & $0.60^{*}$ \\
Lat S' $\left.^{-1} \mathrm{~cm} \cdot \mathrm{s}^{-1}\right)$ & $0.33^{*}$ & 0.13 & 0.24 \\
Mean S' $\left.^{*} \mathrm{~cm} \cdot \mathrm{s}^{-1}\right)$ & $0.49^{*}$ & 0.26 & $0.39^{*}$ \\
Sep E' $\left(\mathrm{cm} \cdot \mathrm{s}^{-1}\right)$ & 0.15 & -0.13 & 0.17 \\
Lat E' $\left(\mathrm{cm} \cdot \mathrm{s}^{-1}\right)$ & 0.10 & 0.04 & -0.02 \\
LVEF $(\%)$ & -0.11 & 0.03 & -0.01 \\
SV $(\mathrm{mL})$ & $0.59^{*}$ & $0.62^{*}$ & $0.64^{*}$ \\
GLS $(\%)$ & -0.09 & 0.05 & -0.14 \\
Circumferential strain $(\%)$ & -0.06 & -0.1 & -0.09 \\
\hline
\end{tabular}

$* \mathrm{P}<0.05$

S' - Systolic longitudinal velocity; E' - diastolic velocity; $\mathrm{VO}_{2}$ - oxygen uptake; LVEF - Left ventricular ejection fraction; SV - stroke volume; GLS - global longitudinal strain

Figure legends:

Figure 1: Mechanical augmentation for S', E', LVEF, GLS throughout exercise

(S' - systolic velocity; E' - diastolic velocity; LVEF - left ventricular ejection fraction; GLS - global longitudinal strain; $\mathrm{VO}_{2}$ - oxygen consumption)

Figure 2: $\mathrm{S}^{\prime}-\mathrm{VO}_{2}$ relationship throughout exercise

( $\mathrm{S}^{\prime}$ - systolic velocity; $\mathrm{VO}_{2}$ - oxygen consumption)

Figure 3: Systolic efficiency slope (SES) - S' $-\mathrm{VO}_{2}$ relationship

(S' - systolic velocity; $\mathrm{VO}_{2}$ - oxygen consumption; sep - septal; lat - lateral) 

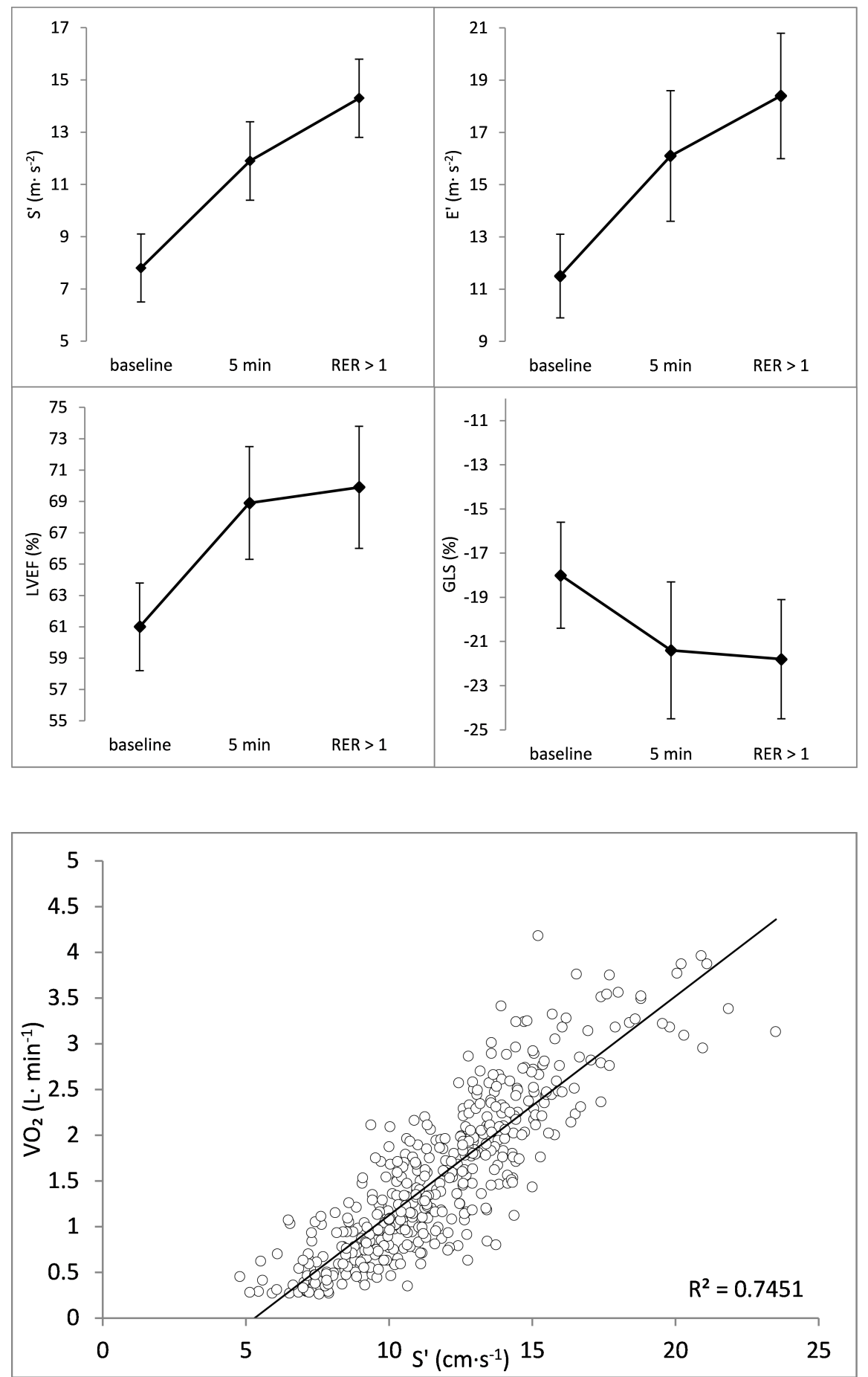


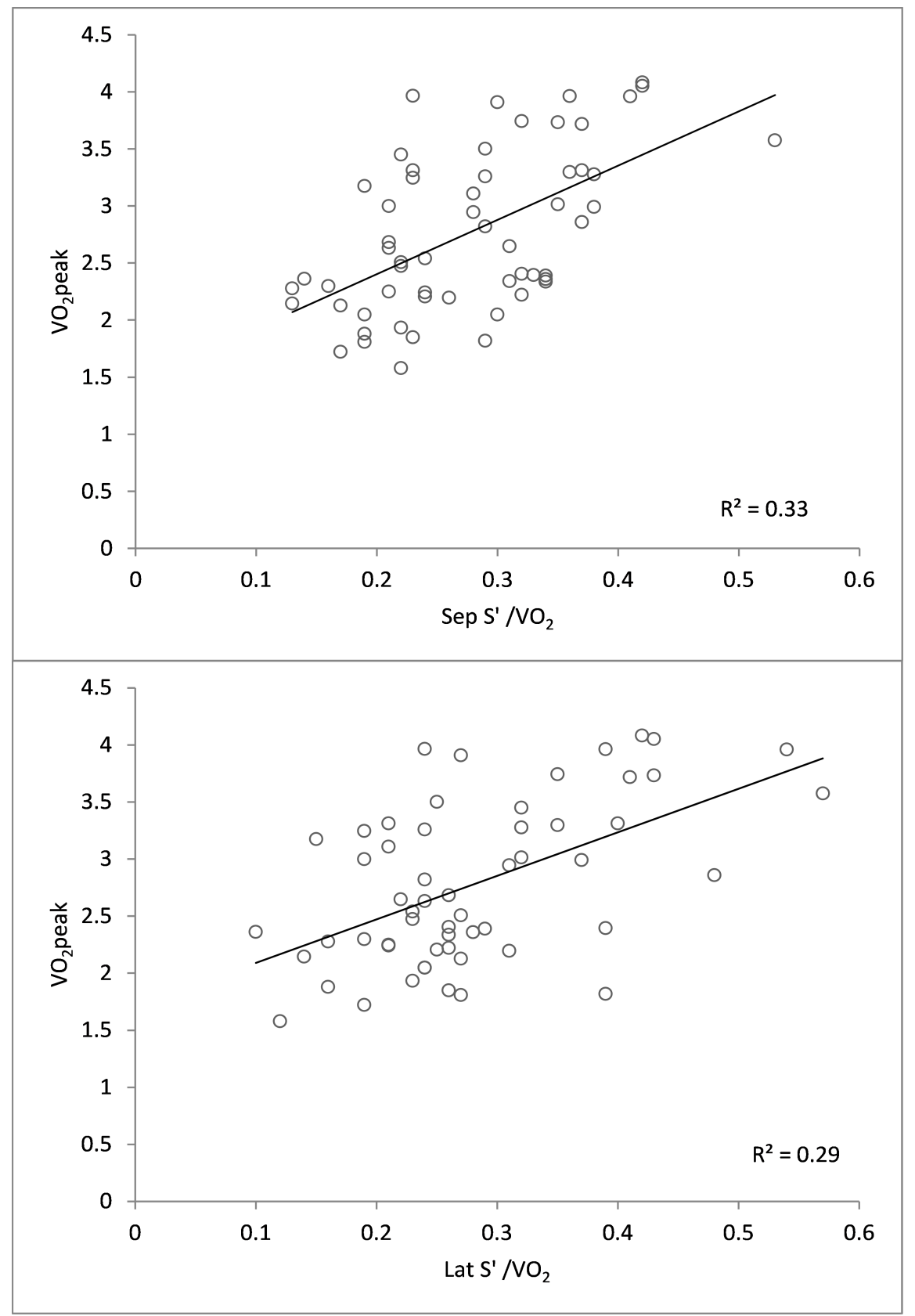

